SLAC-TN-10-042

LCLS-TN-08-1

\title{
Thermal Tests of Undulator Quad
}

\author{
Andrew Fisher
}

$3 / 12 / 2008$

\begin{abstract}
Running at the nominal temperature, the undulator quadrupole has a several degree temperature increase. This not describes the test used to determine the effect on the undulator integrals from the temperature gradient caused by the heat from the quadrupole conducting down the beam pipe.
\end{abstract}

\section{1. Introduction ${ }^{1}$}

The undulator quadrupoles running at their nominal current of 4 amps heat up approximately 4 degrees Celsius; this magnet in turn heats up the beampipe which goes into the undulator. The heating ends up introducing a thermal gradient across the undulator which causes small changes in the magnetic field of the heated poles. By measuring the temperature change in the poles we can model the effects on the field and determine what the magnetic errors will be.

\section{2. Setup}

A test setup was used to simulate undulator heating by the quadrupole. The undulator quadrupole was placed on it's stand, and place a mock up beampipe which was created by using a $\sim 2$ meter tube of aluminium with one end flattened to fit inside the undulator. The aluminium tube was chose to be a "worse case" scenario, as the actual beam pipe should be able to convect more heat away before it starts heating the undulator. Five thermistor probes were placed on the top and bottom poles of the undulator along it's length to acquire the primary measurements. Probes were also placed on the beampipe itself, on the quadrupole, and in 2 locations to measure the ambient temperature. The quadrupole was then run at $\sim 4$ amps for 2-3 days, taking data from all the thermistors every 60 seconds.

\footnotetext{
${ }^{1}$ Work supported in part by the DOE Contract DE-AC02-76SF00515. This work was performed in support of the LCLS project at SLAC.
} 


\section{3. Analysis}

To remove systematic differences in the thermistors, 5 points were taken at the beginning of the run before the power supply was turned on, the average of these five points was then subtracted from the measurements before comparisons were made so that DC offsets in the thermistors would be removed. To find the temperature gradient in the undulator, we compared the 5 poles which had thermistors on them, using an average of the temperature measured by two thermistors one at the top and one at the bottom of the pole. An exponential dependance of temperature with distance was found to be the best fit for these points.

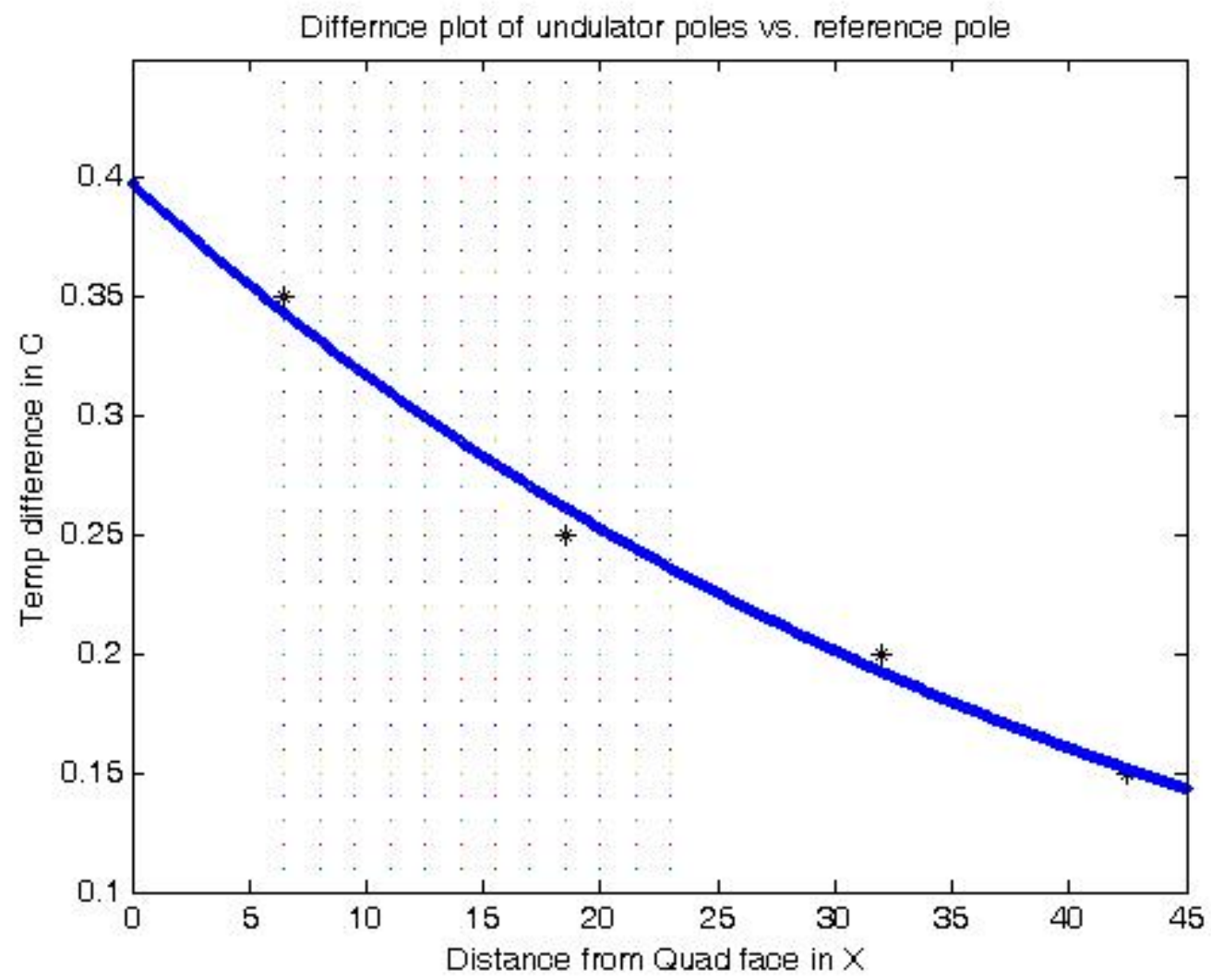

This chart shows the fit of the difference of the temperature at the individual poles from the reference pole. 
Using this fit, it was possible to calculate the approximate temperature change for each pole, and then the error integrals caused by the temperature gradient. These calculations were completed using the following:

$\int B d l=\int_{0}^{z} B\left(z^{\prime}\right) d z^{\prime}$

Using a simplified $\mathrm{B}\left(\mathrm{z}^{\prime}\right)$ this becomes simply

$\sum B_{n} * 1.5 \mathrm{~cm}$

Where $\mathrm{B}_{n}$ is the field at each pole given by

$B_{n}=\Delta T_{n} * B_{\text {pole }} * \frac{.001 T}{1^{\circ} \mathrm{C}}$

Substituting this in you get

$\int B d l=\sum \Delta T_{n} * B_{\text {pole }} * 1.5 \mathrm{~cm} * \frac{.001 T}{1^{\circ} \mathrm{c}}$

To get the second integral, follow the same steps as above to get this equation where $\mathrm{L}$ is the length of the undulator and $\mathrm{Z}$ is the distance from the current position to the end of the undulator

$$
\iint B d l d l^{\prime}=\sum \Delta T_{n} * B_{\text {pole }} * 1.5 \mathrm{~cm} *\left(L-Z_{n}\right) * \frac{.001 T}{1^{\circ} c}
$$

Using these equations we were able to calculate the first and second error integrals for $\mathrm{n}$ terms. The number of terms was chosen by iterative calculating of terms until the integral was unchanging. It was found that the first six poles was sufficient to calculate the first error integral and that the first 12 poles were necessary to calculate the second error integral. Using the fit to determine the temperature at each pole, we found that the integrals were:

$$
\begin{aligned}
& I 1=-6.29766 * 10^{-6} \text { Tesla Meters } \\
& I 2=-1.9 * 10^{-5} \text { Tesla Meters }
\end{aligned}
$$

\section{4. Conclusions}

Although the undulator quad did introduce a temperature gradient across the beam pipe, and through this to the undulator itself, the calculated effect on the first and second integrals of the undulator was below tolerance. This result was very good for a "worse case" setup. It is likely that the actual undulator setup in the tunnel will have an even smaller temperature gradient. 